\title{
Cerebellar Hypoplasia
}

National Institute of Neurological Disorders and Stroke (NINDS)

\section{Source}

National Institute of Neurological Disorders and Stroke (NINDS). Cerebellar Hypoplasia

Information Page.

Cerebellar hypoplasia is a neurological condition in which the cerebellum is smaller than usual or not completely developed. Cerebellar hypoplasia is a feature of a number of congenital (present at birth) malformation syndromes, such as Walker-Warburg syndrome (a form of muscular dystrophy. It is also associated with several inherited metabolic disorders, such as Williams syndrome, and some of the neurodegenerative disorders that begin in early childhood, such as ataxia telangiectasia. In an infant or young child, symptoms of a disorder that features cerebellar hypoplasia might include floppy muscle tone, developmental or speech delay, problems with walking and balance, seizures, intellectual disability, and involuntary side to side movements of the eyes. In an older child, symptoms might include headache, dizzy spells, clumsiness, and hearing impairment. 